Franchir l'infranchissable? Coopération judiciaire et reconnaissance mutuelle dans un espace européen de justice, liberté, et sécurité

\title{
Maik Martin
}

\section{(2) OpenEdition Journals}

Édition électronique

URL : http://journals.openedition.org/conflits/2058

DOI : $10.4000 /$ conflits.2058

ISSN : 1777-5345

Éditeur :

CCLS - Centre d'études sur les conflits lilberté et sécurité, L'Harmattan

Édition imprimée

Date de publication : 1 juin 2006

Pagination : 63-77

ISBN : 2-296-01073-3

ISSN : 1157-996X

Référence électronique

Maik Martin, « Franchir l'infranchissable ? Coopération judiciaire et reconnaissance mutuelle dans un espace européen de justice, liberté, et sécurité », Cultures \& Conflits [En ligne], 62 | printemps 2006, mis en ligne le 19 juillet 2006, consulté le 30 mars 2021. URL : http://journals.openedition.org/conflits/ 2058 ; DOI : https://doi.org/10.4000/conflits.2058

Ce document a été généré automatiquement le 30 mars 2021

Creative Commons License 


\title{
Franchir l'infranchissable? Coopération judiciaire et reconnaissance mutuelle dans un espace européen de justice, liberté, et sécurité
}

\author{
Maik Martin
}

1 L'Union européenne est devenue un espace de «justice, liberté et sécurité » pour ses citoyens ${ }^{1}$. C'est du moins ce que le traité d'Amsterdam de l'Union européenne demande aux Etats membres de développer et ce, à travers une action commune dans le domaine de la coopération judiciaire. Reprenant cette déclaration lors du sommet de Tampere en $1999^{2}$ et du Programme de La Haye en $2004^{3}$, les Etats européens vont adopter et confirmer ces orientations en faisant de la reconnaissance mutuelle la pierre angulaire de la coopération judiciaire. Cette perspective constitue la base juridique d'une pléthore de mesures qui ont été entérinées, ou qui sont actuellement en phase de discussion et de rédaction, dans le domaine de la coopération judiciaire et policière du traité de l'Union, appelé communément « 3e pilier ».

2 En mai 2005, la Commission européenne a considéré que la mise en œuvre du principe de reconnaissance mutuelle représentait l'une des perspectives les plus prometteuses dans le domaine pénal ${ }^{4}$. Au-delà de la discussion sur le bien-fondé d'une telle affirmation, indubitablement l'ensemble des promesses associées au principe de reconnaissance mutuelle pourra difficilement être mis en œuvre in praxi. Nous évoquerons dans cet article les principales orientations jurisprudentielles qui ont été définies à partir des premières affaires traitées par les Cours anglaises, irlandaises et allemandes, concernant la mise en œuvre juridique du mandat d'arrêt européen $(\mathrm{MAE})^{5}$, premier instrument de reconnaissance mutuelle entré en vigueur. Nous démontrerons, à travers ces expériences judiciaires, que la perspective faisant de la reconnaissance mutuelle l'un des facteurs positifs centraux de l'espace pénal européen 
est discutable. L'application cohérente et effective du principe de reconnaissance mutuelle nécessite, en réalité, l'assurance d'un certain nombre de règles juridiques essentielles, comme la protection adéquate des droits fondamentaux des individus, l'harmonisation législative des procédures judiciaires nationales, en particulier concernant les procédures de poursuites, ainsi qu'un échange entre les autorités judiciaires des Etats membres. Bon nombre des difficultés qui sont apparues au sein des Cours anglaises, irlandaises et allemandes dans l'application du MAE résident dans l'absence et le degré d'harmonisation insuffisant des législations pénales au niveau des droits et des procédures, mais aussi dans le manque évident de relation de confiance indispensable entre les autorités judiciaires européennes sur les normes de chaque système.

Reconnaissance mutuelle et Programme de La HayeLe principe de reconnaissance mutuelle prévu par le Conseil européen

3 Le concept de reconnaissance mutuelle, qui est au cœur des efforts de l'Union européenne pour renforcer la coopération judiciaire, est l'un des principes largement reconnus et mis en valeur par le système juridique européen ${ }^{6}$. Depuis l'arrêt Cassis de Dijon ${ }^{7}$ rendu par la CJCE, la réglementation européenne sur le marché intérieur affirme la libre circulation, au sein de la Communauté, des marchandises commercialisées dans les Etats membres. Du fait des Conventions européennes relatives aux procédures civiles, et en particulier celle du Conseil européen (CE) n44/2001 concernant la reconnaissance et l'exécution des jugements civils et commerciaux ${ }^{8}$, les tribunaux des Etats membres sont tenus de reconnaître et de faire appliquer dans leurs juridictions, de façon automatique et sans modification, les décisions finales des autres Cours européennes.

4 A la suite des conclusions de Tampere de 1999 et conformément au Programme de La Haye de 2004, ce principe doit former le cœur juridique de la coopération en matière civile, mais aussi pénale. Cette orientation a provoqué des évolutions significatives visà-vis des voies classiques de coopération judiciaire. Jusqu'ici, un grand nombre de traités bilatéraux ou multilatéraux (notamment la Convention européenne d'entraide judiciaire de 1959) ${ }^{9}$ avait été établis à partir du principe d'entraide judiciaire. Cela signifiait que les Etats devaient adresser à l'Etat requis une demande pour la poursuite judiciaire d'une personne ou pour l'exécution d'une décision judiciaire, en l'accompagnant de preuves suffisantes. Les Cours et tribunaux de l'Etat requis statuaient alors de façon autonome sur le fait d'exécuter la demande d'entraide (et, particulièrement dans les affaires d'extradition dans lesquelles la dimension politique est importante). Si l'on admet que les conclusions des Conventions européennes du Conseil de l'Europe ${ }^{10}$ s'inscrivent largement en faveur de la coopération, les Etats signataires ont néanmoins largement réduit les possibilités d'octroi d'entraide ou d'extradition des personnes, écartant toute forme d'automaticité. Enfin, l'entraide au niveau pénal ne pouvait être mise en œuvre uniquement si le principe de double incrimination - reconnaissance de l'infraction par le droit pénal de l'Etat requérant et de l'Etat requis - était applicable.

5 Aujourd'hui, le principe de reconnaissance mutuelle adopté par les Etats européens entraîne la construction d'une nouvelle approche, en raison de la forte réduction des possibilités offertes à l'Etat requis de refuser l'exécution de la demande de l'Etat émetteur, et de l'évolution de l'exigence de double incrimination. En réduisant ainsi le pouvoir de décision de l'Etat saisi, à travers l'établissement d'une reconnaissance et 
d'une exécution automatique des décisions étrangères, le Conseil européen et la Commission européenne ont soutenu une telle évolution. Le Conseil européen décrit de façon euphorique cette nouvelle étape comme la libre circulation des décisions juridiques au sein de l'Union européenne ${ }^{11}$. A partir des perspectives ambitieuses ouvertes par les conclusions de Tampere et du Programme de La Haye, le Conseil européen a, à l'heure actuelle, adopté trois décisions-cadres mettant en jeu ce principe de reconnaissance mutuelle dans le domaine de la coopération judiciaire : la décisioncadre relative à la mise en œuvre du $\operatorname{MAE}^{12}$, la décision-cadre relative à la reconnaissance mutuelle des délits financiers ${ }^{13}$, et la décision-cadre relative au gel des biens et des avoirs ${ }^{14}$. De nouvelles mesures sont actuellement en cours de discussion. Elles concernent l'ensemble des étapes de la procédure pénale, comme le recueil et la transmission des preuves, la reconnaissance des infractions supplémentaires commises dans un autre Etat membre, ou encore le transfert des personnes dans la phase de préparation et d'exécution du jugement ${ }^{15}$. La plupart de ces instruments se structurent à partir de trois caractéristiques qui symbolisent le principe de reconnaissance mutuelle dans la coopération judiciaire européenne.

6 La première caractéristique est le devoir pour l'Etat saisi d'exécuter la décision qu'une autorité judiciaire d'un autre Etat membre lui aurait transmise, en conformité avec un certain nombre d'exigences formelles présentes, par exemple, dans le «formulaire préétabli » du MAE contenu dans la décision-cadre européenne. Un MAE doit, alors, contenir des informations concernant la personne recherchée, la procédure ouverte contre cette dernière, une description des éléments juridiques et des preuves accusatoires, ainsi que les garanties d'un jugement et d'une poursuite exécutoire dans l'Etat requérant ${ }^{16}$. Idéalement, l'autorité requise ne doit pas aller au-delà des informations prévues par le formulaire et les différentes cases à remplir. A prima facie, une affaire regroupant l'ensemble des preuves nécessaires à l'application de la reconnaissance et de l'exécution de la demande ne pourra pas être rejetée par l'Etat requis. Ainsi, une fois que les différentes lignes et cases essentielles ont été remplies et que la demande d'information a été transmise, le MAE doit prendre effet. L'Etat requis, en règle générale, ne peut pas refuser de façon discrétionnaire de reconnaître et d'exécuter la décision étrangère. Cette procédure, marquée par une logique d'automaticité via l'utilisation d'un "formulaire préétabli», constitue la base du système de libre circulation des décisions judiciaires au sein de l'Union européenne ${ }^{17}$.

7 La deuxième caractéristique est la réduction radicale des raisons laissées à l'Etat requis pour justifier un refus de reconnaître et d'exécuter la demande étrangère. Précisément, dans le cas de la décision-cadre relative au MAE, les possibilités de refus sont limitées aux cas d'amnistie dans l'Etat requérant; de risque d'une non-application du principe de non bis in idem qui sous-entend l'impossibilité pour une personne d'être accusée dans un autre pays d'un délit déjà jugé ; de non-respect du délai de prescription dans l'Etat requis ; ou encore, de non-respect du principe de territorialité à travers une demande concernant un délit commis en réalité dans l'Etat d'exécution ${ }^{18}$.

8 Enfin, le troisième et probablement le plus controversé des éléments constitutifs du principe de reconnaissance mutuelle, est l'évolution de l'obligation de la double incrimination. Les implications d'un tel changement seront discutées dans la suite de cet article.

Des mesures-cadres : une question de confiance mutuelle 
9 Dans la logique reconnue par le Conseil européen à travers les conclusions de Tampere et le Programme de La Haye, les mesures obligeant un Etat membre à reconnaître et à exécuter un jugement provenant d'une juridiction étrangère doivent répondre à une exigence et à un consensus sur l'harmonisation des droits procéduraux pour les personnes affectées par les décisions judiciaires. Comme l'ont envisagé le Conseil européen et la Commission européenne, cette évolution ne peut alors se concrétiser que s'il existe un degré raisonnable de confiance et d'échange entre les Cours nationales. Par la transmission d'un formulaire d'extradition, les autorités judiciaires doivent accepter une restriction de leurs compétences et de leurs contrôles sur les décisions d'exécution et d'application émanant d'un autre Etat membre ${ }^{19}$.

10 L'idée de faire de la confiance l'élément permettant le renforcement d'une culture judiciaire commune a été diffusée par la Commission européenne ${ }^{20}$ avec l'adoption et la mise en œuvre d'instruments de coopération dont l'objectif est d'« étoffer " les droits évoqués par l'article 6 de la Convention européenne des droits de l'Homme ${ }^{21}$ et par la jurisprudence de la Cour européenne de Strasbourg. Ce projet a été l'objet d'une proposition de décision-cadre ${ }^{22}$ de la part de la Commission, fixant les garanties pour le respect des droits de la défense tels l'accès à un avocat, à un interprète et la garantie d'une aide juridique. Cette proposition est actuellement en débat au sein du Conseil européen, où il semble que les discussions, concernant un accord sur la portée, le contenu et les relations avec la Cour européenne des droits de l'Homme, soient difficiles. Selon la Commission et le Conseil européen ${ }^{23}$, l'établissement de normes communes sur la présomption d'innocence et l'admission des preuves doit représenter la base nécessaire à la réalisation d'une libre circulation des décisions judiciaires dans l'espace européen. Cependant, si l'harmonisation ou la mise en conformité des règles nationales sont des mesures essentielles pour insuffler un certain degré de confiance entre les systèmes judiciaires des Etats, cela ne suffit pas à constituer un véritable dialogue entre les autorités pénales européennes. L'adhésion à une culture judiciaire commune semble passer par l'acceptation d'une haute exigence concernant les droits procéduraux et la réciprocité entre les représentants judiciaires. La place laissée à la sauvegarde des droits des suspects dans l'harmonisation des différents systèmes nationaux constitue une étape fondamentale dans la concrétisation pratique d'une réelle coopération judiciaire.

La construction d'un espace judiciaire européen : la question des garanties procédurales et des droits fondamentaux

11 A la vue des premiers jugements des autorités judiciaires nationales concernant la mise en œuvre du premier véritable instrument de reconnaissance mutuelle que constitue le MAE, la formation de l'espace judiciaire européen doit faire face à des résistances dans le domaine de la reconnaissance et de l'exécution des décisions entre Etats membres. Si les arrêts des Cours anglaises, irlandaises et allemandes constituent la preuve que l'adoption de mesures au niveau européen développe les échanges et la confiance entre les juridictions nationales, un dispositif comme le MAE reste encore en avance sur les pratiques réelles ${ }^{24}$. Les décisions énoncées par les Cours nationales démontrent clairement que la question de la reconnaissance mutuelle entre les systèmes pénaux de chaque pays européen s'inscrit dans des considérations et des prises de position complexes, empêchant la mise en place d'une solution claire. Différents juges se sont intéressés au principe de reconnaissance mutuelle et à la limitation que ce dernier entraîne sur leur pouvoir d'investigation d'une part, via l'examen des procédures 
étrangères relatives aux infractions constatées ou supposées (en particulier celles concernant l'exécution) et d'autre part, à travers le respect des droits de la défense. Une approche cohérente des logiques judiciaires en Angleterre et en Irlande n'est pas encore apparue concernant cette problématique, car comme nous le démontrerons, la jurisprudence de ces Cours en est encore au stade expérimental.

Le « formulaire d'extradition préétabli » : entre confiance et domaine de compétence

A propos de ce que nous avons nommé « le formulaire préétabli » du MAE - qui se doit d'être adopté par les Cours des Etats membres requis - une interrogation essentielle a émergé, focalisant un grand nombre de discussions au sein des autorités judiciaires nationales: dans quelle mesure les Cours chargées de l'exécution d'un MAE peuventelles statuer sur la validité d'une telle demande juridique, de sa conformité vis-à-vis de la décision-cadre européenne et de ses mises en œuvre dans les législations nationales ? Il est arrivé qu'on s'interroge au cours de certaines décisions pour savoir si les juridictions saisies ont le pouvoir, ou même la compétence d'examiner les faits commis et décrits dans le MAE par les autorités requérantes conformément au formulaire préétabli. Devant l'absence de normes communes, les Cours se sont intéressées à cette problématique. Dans le cas Palar v Court of First Instance Brussels ${ }^{25}$, la High Court ${ }^{26}$ anglaise a refusé l'exécution d'un mandat européen transmis par les autorités belges, déclarant que si la Cour se doit d'inscrire sa décision dans une logique de coopération et de discussion avec les autorités judiciaires des autres Etats membres, cela ne doit pas être fait au détriment de l'exigence d'une transmission correctement garantie du délit d'extradition. Dans la même logique, la Cour d'appel allemande de Karlsruhe a souligné qu'il était nécessaire de satisfaire au respect de double incrimination prévue par le MAE en fonction de la législation de l'Etat requérant ${ }^{27}$. Cependant, dans le cas Office of the King's Prosecutor Brussels v Cando Armas \& ors ${ }^{28}$, Lord Hope of Craighead a estimé, à la chambre des Lords, que les juges devaient s'assurer que le délit présumé et exposé dans le mandat était en conformité avec les exigences nécessaires à l'extradition conformément aux dispositions de l'Acte d'extradition anglais qui met en œuvre la décision-cadre européenne. En conséquence, les juges ne doivent pas prendre en compte le fait que ce délit soit passible d'extradition dans la législation du pays requérant. Un raisonnement similaire a été utilisé par la juge Smith dans le cas Boudhiba v Central Examining Court $\mathrm{n}^{\circ} 5$ of the National Court of Justice Spain ${ }^{29}$, s'appuyant sur l'ethos de confiance à la base de l'existence du MAE, au sens où les Etats membres n'émettraient que des MAE qui soient en accord avec leurs propres normes.

Dans une logique quelque peu contradictoire, les Cours irlandaises se sont, elles aussi, interrogées sur les compétences des autorités requises concernant l'examen de la validité des procédures ayant abouti à l'émission d'un mandat d'arrêt européen. Dans l'affaire Minister for Justice, Equality and Law Reform v Fallon ${ }^{30}$, le juge Geoghegan, de la High Court irlandaise, s'est déclaré compétent pour rejeter un mandat émis par les autorités anglaises, en raison du non-respect des garanties nationales, avant même l'édition de cette demande. En estimant, de façon générale, que la Cour devrait s'abstenir de statuer sur la validité de l'extradition à moins que celle-ci ne respecte pas les droits fondamentaux et constitutionnels, le juge a considéré que le mandat émis par les autorités anglaises devait être invalidé, et a ainsi rejeté la demande de remise de la personne poursuivie. Dans une logique comparable, la Cour d'appel de Stuttgart ${ }^{31}$ a tenu à affirmer, et cela malgré l'absence de dispositions relatives à la limitation dans le temps des procédures dans le système du pays émetteur, que les autorités judiciaires 
nationales devaient être compétentes et ainsi mandatées pour l'exécution concrète du mandat, en particulier concernant les enquêtes sur les délais de procédure et leur respect dans le pays requérant. Néanmoins, dans l'affaire irlandaise Fallon v Governor of Cloverhill Prison ${ }^{32}$, la Cour suprême irlandaise a cette fois considéré que l'exécution intégrale du MAE pouvait être remise en cause seulement en cas de manquement exceptionnel et de mauvaise foi avérée.

Enfin, un cas très intéressant se doit d'être présenté : l'affaire Enander v Governor of HMP Brixton and the Swedish National Police Board ${ }^{33}$, dans laquelle la High Court anglaise devait statuer afin de savoir si un mandat européen émis par le Conseil national de la police suédoise pouvait être considéré comme une demande transmise par " une autorité judiciaire " au regard de la loi d'extradition britannique de 2003 et de la décision-cadre européenne. Sous l'article 6 de cette décision-cadre, la désignation de l'autorité juridique compétente est laissée aux autorités des Etats membres, la seule exigence étant la validation de cette instance par le Conseil européen. Le législateur suédois a officiellement désigné le Conseil de la police comme autorité compétente pour émettre des MAE. En conséquence, la Cour britannique a donc refusé d'aller dans le sens du mandat européen et du choix suédois de l'autorité compétente, alors même que la décision-cadre n'avait pas défini le terme d'«autorité judiciaire» et avait laissé expressément le libre choix à chaque Etat. Comme il a été souligné par l'un des juges dans cette décision, toutes autres interprétations exigeraient que chaque Etat d'exécution examine si la fonction de l'autorité qui a émis le mandat peut être reconnue comme une autorité juridique au niveau du système pénal britannique. En allant ainsi à l'encontre du principe de reconnaissance et de confiance mutuelle sous-entendu dans la décision-cadre, cette orientation jurisprudentielle introduit une difficulté supplémentaire au niveau de l'efficience et de l'efficacité du mandat d'arrêt européen.

16 Tous ces jugements illustrent les difficultés rencontrées par les Cours nationales pour mettre en œuvre juridiquement le concept de reconnaissance mutuelle dans une logique de confiance et de respect des procédures criminelles étrangères. La décisioncadre relative au mandat d'arrêt européen n'aborde, par ailleurs, pas la question de l'étendue des compétences des autorités judiciaires requises pour se saisir d'un mandat émis sous la forme prescrite par le formulaire préétabli. Les juges chargés de l'exécution doivent alors adopter une approche cohérente et claire, quant à savoir jusqu'où et comment ils peuvent chercher et poursuivre leur enquête sur la conformité et la validité d'un MAE vis-à-vis des lois de l'Etat requérant. Ces difficultés vont probablement être renforcées lorsque les Cours devront faire face aux défis de l'exécution de pénalités financières ou de sentences de prison émises par un autre Etat membre, conformément aux futurs instruments du 3e pilier.

La question du respect de la Convention européenne des droits de l'Homme et de la reconnaissance mutuelle

17 Si les cas précédemment cités abordent essentiellement les questions de validité et de conformité procédurales des MAE vis-à-vis du principe de la reconnaissance mutuelle, le lien entre reconnaissance et confiance mutuelle au sein des systèmes juridiques pénaux respectifs doit être approfondi. Cette relation est d'autant plus cruciale dans les cas où l'application de la décision juridique étrangère porterait atteinte aux droits fondamentaux de la personne visée. Alors qu'il est défini qu'une personne sous le coup d'un mandat d'arrêt européen ne peut pas être extradée dans l'Etat requérant si le 
procès ne répond pas aux exigences de l'article 6 de la Convention européenne des droits de l'Homme, comment les Cours nationales chargées de l'exécution vont-elles traiter ces obligations dans le domaine de la reconnaissance mutuelle et de sa mise en œuvre juridique?

La décision-cadre du MAE ne contient pas expressément de dispositions permettant le refus d'exécution d'un mandat d'extradition pour non respect des droits de l'Homme. Néanmoins, dans ses articles 1(3), 12 et 13, le dispositif européen garantit les droits fondamentaux et ne modifie en aucun cas l'obligation qui est faite aux Etats membres de respecter ces derniers. Cependant, des Etats comme l'Allemagne, le Royaume-Uni et l'Irlande ont, dans la mise en œuvre de l'instrument, donné un effet juridique à ces clauses, en stipulant expressément des motifs permettant le refus d'exécution d'un mandat d'arrêt européen pour non-respect des droits de l'Homme ${ }^{34}$.

C'est dans cette logique que les Cours anglaises et irlandaises ont mis en pratique ces clauses dans le traitement du MAE, ainsi que dans d'autres affaires d'extradition où ont surgi ces interrogations. Il est difficile en quelques lignes de résumer l'ensemble des cas, dont les orientations ont été hétérogènes et inconstantes. Une question reste encore ouverte : quelle importance les autorités judiciaires attachent-elles au fait que les Etats requérants sont des signataires de la Convention européenne des droits de l'Homme et quel est le degré de confiance à attacher à une telle adhésion? Au Royaume-Uni, les Cours saisies des affaires d'extradition ont suivi le précédent établi par la chambre des Lords dans le cas de la demande d'asile $\mathrm{R}$ (on the application of Ullah) v Special Adjudicator ${ }^{35}$. Dans cette décision, la chambre des Lords a estimé que la référence à la Convention européenne des droits de l'Homme, et notamment à son article 3, ne pouvait être invoquée que si l'on pouvait clairement démontrer que les droits en question avaient été bafoués et non respectés dans l'enquête menée par l'Etat demandeur. La High Court anglaise dans son arrêt de non-exécution du MAE, dans l'affaire R (on the application of Bermingham) v Director of the Serious Fraud Office, a expressément adopté cette approche ${ }^{36}$. Cette position a également été suivie dans l'affaire Boudhiba v Central Examining Court $n^{\circ} 5$ of the National Court of Justice Spain. La juge Smith, de la High Court anglaise, a estimé que même s'il y avait des preuves anecdotiques quant aux infractions aux droits de l'Homme dans le pays requérant, le terroriste présumé d'al-Qaida qui était recherché par l'Espagne, pouvait néanmoins être extradé, puisqu'il lui était possible, si nécessaire, de porter la question du nonrespect de ses droits auprès des Cours espagnoles et de la Cour européenne de Strasbourg. Dans ces conditions, une demande de mandat d'arrêt européen ne peut être refusée pour des raisons de non-respect des droits de l'Homme que dans le cas où la personne recherchée peut prouver que son extradition conduirait à un danger réel quant au respect de ses droits ${ }^{37}$. En ce qui concerne la question de la longueur de la détention préventive, la juge a refusé de considérer ce qu'elle décrit comme une disposition légale de loi espagnole. Selon elle, en absence de disposition concernant la longueur des détentions provisoires au niveau de la Cour de Strasbourg, l'infraction de l'article 5 de la Convention européenne des droits de l'Homme ne peut être évoquée. Cette logique de confiance assumée envers la Convention européenne des droits de l'Homme et la capacité compensatrice de la Cour de Strasbourg a été suivie de façon similaire par les High Courts anglaises dans les affaires Ramda v Secretary of State for the Home Department and the Government of France ${ }^{38}$ et $R$ (on the application of Labi) v Secretary of State for the Home Department ${ }^{39}$. 
20 A l'inverse, dans le premier appel de l'affaire Ramda v Secretary of State for the Home Department ${ }^{40}$ de 2002, la High Court anglaise a fait remarquer que la Cour européenne des droits de l'Homme n'était pas une cour d'appel, comme elle l'a elle-même indiqué, soulignant ainsi que la compétence pour juger de la conformité vis-à-vis de la Convention européenne des droits de l'Homme et des réparations en cas de non-respect revenait aux Cours nationales. Ainsi, la High Court n'a pas suivi les justifications des autorités britanniques dans leur idée de considérer la Cour de Strasbourg comme un élément du système pénal français, permettant le respect des droits d'une personne à un procès équitable après son extradition en France. Dans le cas de l'affaire Minister for Justice, Equality and Law Reform v Stapleton ${ }^{41}$ traitée par la High Court irlandaise, le juge Peart est allé encore plus loin, en affirmant que si la Cour pouvait se satisfaire de certaines exigences présentes au niveau de l'Etat requérant concernant un procès équitable envers la personne recherchée, elle était néanmoins tenue par les clauses relatives aux droits de l'Homme contenues dans l'Acte irlandais de mise en œuvre du MAE de 2003. Dès lors, le juge se déclare compétent pour refuser la remise de la personne recherchée et ce, malgré l'existence, au sein de l'Etat requérant, de dispositifs garantissant le respect d'un procès équitable. Il est alors explicitement exposé que la personne recherchée pourra bénéficier des droits garantis par la Constitution irlandaise et l'Acte susmentionné, plutôt que de ceux proposés par l'Etat requérant.

21 La question du refus de la reconnaissance et de l'exécution d'un MAE - premier instrument de mise en œuvre de la reconnaissance mutuelle - en raison du non-respect constaté ou présumé de la Convention européenne des droits de l'Homme est donc encore loin d'avoir été résolue. Il sera toujours légitime de se demander jusqu'à quel point les Cours nationales, devant statuer sur une décision juridique étrangère, peuvent faire confiance à un Etat membre du fait de son adhésion à la Convention européenne des droits de l'Homme et de l'efficacité des mécanismes de Strasbourg.

Une telle interrogation est complexe, dans le sens où elle s'inscrit dans la discussion du respect des normes de la Convention européenne des droits de l'Homme par les Etats membres de l'Union européenne. Les Cours d'exécution exigent qu'un suspect établisse un degré flagrant et irréparable de violations de la Convention européenne des droits de l'Homme au niveau du pays requérant. Dès lors, l'exécution d'un mandat d'arrêt européen serait très certainement limitée par une atteinte irrémédiable aux droits fondamentaux des personnes, ce qui aurait, dans certains cas, un très grand pouvoir d'intimidation. On doit concéder que cette question ne représente pas un obstacle uniquement au niveau du mandat d'arrêt européen, mais qu'elle correspond plus largement à l'exécution de l'obligation de reconnaissance mutuelle entre les Etats membres. Il s'agit d'un problème général d'évaluation des violations des droits fondamentaux dans un pays tiers, dans une perspective ex ante, ce qui pose la question de la légitimité d'orienter une victime potentielle de telles violations vers un mécanisme ex post facto de recours vers une autre juridiction, avec la possibilité de saisir une autorité internationale. Cependant, l'adoption au sein du troisième pilier d'instruments permettant de garantir le respect des normes procédurales et étoffant ainsi les droits prévus par l'article 6 de la Convention européenne des droits de l'Homme, conduirait à rendre plus facilement invocables et applicables de telles règles juridiques. La mise en œuvre d'un tel instrument n'empêcherait pas, bien évidemment, les risques d'infractions vis-à-vis des normes fondamentales. Il constituerait, en réalité, une nouvelle base juridique à partir de laquelle pourrait se renforcer la confiance 
mutuelle entre les autorités pénales des divers systèmes européens. Dans son rapport relatif aux droits procéduraux dans les poursuites en matière pénale, la Commission sur l'Union européenne de la chambre des Lords a clairement identifié les difficultés rencontrées par le principe de reconnaissance mutuelle à l'échelle européenne : «Un juge national peut être contraint d'appliquer une décision d'une Cour étrangère sans pouvoir examiner les faits et en se fondant uniquement sur un formulaire dont un certain nombre de cases ont été préalablement cochées. Pour qu'un tel système puisse être acceptable, on doit pouvoir garantir que l'individu au cœur des procédures soit traité avec équité. Il est pour ce faire nécessaire que les Etats membres respectent les standards minimums d'investigation et de poursuites pénales ${ }^{42} »$.

Souveraineté et valeurs communes : vers une harmonisation du droit positif?

Le principe de reconnaissance, tel qu'il apparaît à travers les conclusions de Tampere et le programme de La Haye, illustre les différences qui caractérisent les systèmes judiciaires nationaux. Cette non-harmonisation ne devrait pas bloquer le mouvement de libre circulation des décisions pénales, à condition qu'un certain nombre de normes et de garanties soient incorporées dans les dispositifs judiciaires. Le principe de reconnaissance mutuelle à l'européenne ne se limite pas, cependant, à cette dimension. L'abolition de l'exigence de la double incrimination, qui était traditionnellement incluse dans les Conventions d'entraide pour la quasi-majorité des incriminations communes (meurtre, blessures graves, viol, vol, trafic de drogue, etc.), fait que le droit positif de l'Etat d'exécution ne constitue pas un élément essentiel face à la reconnaissance et à l'application d'une décision émise par un autre Etat membre. A travers la mise en place d'une longue liste de délits qualifiés de "génériques", les différents instruments de reconnaissance mutuelle impliquent que le délit soit répréhensible dans le pays demandeur. La non-criminalisation de certains actes dans la législation du pays requis n'est pas indispensable.

Une telle logique répressive pourrait s'inscrire dans la continuité d'un phénomène d'intégration toujours plus important au niveau européen. C'est, en effet, ce qu'a souligné, dans la chambre des Lords, Lord Bingham de Cornhill concernant l'affaire Cando Armas ${ }^{43}$, à propos de la liste des délits contenus dans la décision-cadre européenne pour lesquels la double incrimination n'est plus nécessaire. Supposant que les délits concernés figurent dans les différents codes pénaux nationaux, Lord Bingham de Cornhill a jugé que la double incrimination pourrait, de fait, aller de soi. Cependant, si une telle supposition était justifiée, l'abolition de l'exigence de double incrimination perdrait son sens. Si la plupart des délits concernés par la fin de la double incrimination sont en effet présents dans les différents codes pénaux des Etats membres, il existe certaines exceptions importantes. Il est nécessaire de rappeler que les définitions de certaines incriminations varient très fortement selon les Etats membres. L'euthanasie, par exemple, tombe généralement dans la définition de meurtre conformément à la loi anglaise et allemande, ce qui, de façon générale, n'est pas le cas pour les Pays-Bas, où cette conduite est couverte par des dispositions spécifiques. Concernant les incriminations de racisme et de xénophobie, qui constituent l'une des catégories retenues par la décision-cadre européenne, la double incrimination est supprimée, alors même que de nombreux cas restent non reconnus par certains Etats. Si le délit de «négationnisme » est expressément inscrit dans le code pénal français, allemand et autrichien, il n'en va pas de même pour la loi anglaise. Dans ces conditions, l'exemple tristement célèbre de David Irving ${ }^{44}$ illustre cette tension. $\mathrm{Si}$ ce dernier avait fait l'objet d'un MAE de la part des autorités autrichiennes, et cela, 
même si sa conduite ne constitue pas un délit dans la loi anglaise, les Cours britanniques auraient tout de même été obligées d'ordonner sa remise aux autorités autrichiennes. De façon plus étonnante, et dans la logique des propositions qui sont actuellement faites ${ }^{45}$ dans le cadre du renforcement de la coopération judiciaire européenne au niveau de l'exécution des peines, les autorités britanniques pourraient, jusqu'à un certain point, être tenues par les décisions judiciaires autrichiennes d'exécuter une peine de prison envers M. Irving. On ne s'étonnera pas alors que cette problématique soit discutée actuellement en Belgique au niveau de la Cour d'arbitrage. Dans une décision relative à la mise en œuvre de la décision-cadre européenne, la Cour d'arbitrage pose ainsi la question préjudicielle à la Cour de Justice de la Communauté européenne, de la compatibilité entre la suppression du devoir de double incrimination et le traité de l'Union européenne et plus largement avec le principe de légalité en matière pénale ${ }^{46}$.

Il est donc difficile de considérer que l'abolition unilatérale de l'exigence de double incrimination constitue une étape vers un renforcement de la confiance mutuelle entre les systèmes judiciaires des Etats membres. Il s'agit plutôt, en réalité, d'un recul, qui obligerait un Etat, ayant fait le choix de ne pas pénaliser de tels comportements, à définir ces derniers, même indirectement, comme des délits criminels sous la pression de la législation d'un autre Etat membre. Un tel effet du principe de reconnaissance mutuelle est en réalité la conséquence de la carence et de l'approximation du processus d'harmonisation du droit positif à l'échelle européenne. Le succès relatif du programme de reconnaissance mutuelle dans le domaine des affaires civiles et commerciales n'était pas aussi dépendant de l'harmonisation des droits positifs européens. Cela doit se comprendre en grande partie du fait de la différence entre les perceptions relatives au droit pénal et au droit privé. Plus que ce dernier, le droit pénal est considéré comme un objet porteur des valeurs sociales et morales dominantes dans une société et encadrées par des normes législatives dans l'exercice de la souveraineté de cet Etat. Il y aurait ainsi beaucoup moins d'empressement à accepter l'intrusion de positions morales incarnées par le système pénal d'un autre Etat, particulièrement lorsque cela concerne des questions telles que l'euthanasie, l'avortement ou le négationnisme. Cette volonté de ne pas subir les orientations pénales des divers Etats européens s'avère un obstacle insurmontable dans le processus d'harmonisation des législations criminelles, malgré l'élan important que cela donnerait au principe de reconnaissance mutuelle ${ }^{47}$.

Confiance, expérience et harmonisation

Au travers d'exemples précis relatifs aux premières décisions concernant l'application du principe de reconnaissance mutuelle via le MAE, cet article a essayé de démontrer que la concrétisation d'un espace judiciaire européen dépendait de la confiance entre les autorités judiciaires des Etats membres sur les normes appliquées par tous. Le renforcement d'une harmonisation des droits procéduraux et positifs des systèmes nationaux faciliterait la reconnaissance mutuelle, en favorisant un dialogue et une confiance entre les représentants judiciaires. Cependant, cette relation de confiance ne peut être ni forcée, ni commandée par des institutions européennes ou par des législations. Il semble que ce soit plutôt à travers des expériences concrètes entre les Cours nationales et le renforcement des obligations de protection des droits et des garanties procédurales, qu'un tel processus puisse se mettre en place. La reconnaissance mutuelle ne représente donc pas la pierre angulaire, mais seulement les briques qui permettront la construction de l'espace de "justice, liberté et sécurité ». Cependant, sans le ciment que constitue la confiance entre les autorités nationales, en 
particulier au niveau des garanties procédurales, l'architecture d'un tel espace ne pourra être stable.

\section{NOTES}

1.Traduction de l'anglais par Antoine Mégie.

2.Conclusions du Conseil européen de Tampere, 15-16 octobre 1999 : http:// www.europarl.eu.int/summits/tam_fr.htm.

3.Conseil de l'Union européenne, 5 novembre 2004, 14292/04 : http://europa.eu.int/ comm/justice_home/doc_centre/doc/hague_programme_en.pdf

4.. Décision-cadre du Conseil, du 13 juin 2002, relative au mandat d'arrêt européen et aux procédures de remise entre Etats membres, 2002/584/JAI, Journal officiel L $190 \mathrm{du}$ 18.07.2002.

5.. Dans la suite de l'article, le mandat d'arrêt européen sera également évoqué par le sigle « MAE ». Communication sur la reconnaissance mutuelle des décisions judiciaires en matière criminelle et sur le renforcement de la confiance mutuelle entre les Etats membres, 19 mai 2005, $\operatorname{COM}(2005) 195$ final, § 4 :

http://europa.eu.int/comm/justice_home/doc_centre/criminal/doc/com_2005_195_en.pdf 6.. Pour une analyse complète du principe de reconnaissance mutuelle, voir Peers $\mathrm{S}$., Mutual Recognition and Criminal Law in the European Union : Has the Council got it Wrong ? (2204) 41 CMLR 5.

7.. Case 120/78 Rewe-Zentral AG v Bundesmonopolverwaltung für Branntwein [1979] ECR 649.

8.. JO L $12 / 1$ 16.01.2001.

9.. Case 120/78 Rewe-Zentral AG v Bundesmonopolverwaltung fr Branntwein [1979] ECR 649.

10.. http://conventions.coe.int/treaty/en/Treaties/Html/030.htm

11.. Préambule 5 de la décision-cadre relative au MAE, op. cit.

12.. Décision-cadre relative au mandat d'arrêt européen, op. cit.

13.. Décision-cadre du Conseil, du 24 février 2005, concernant l'application du principe de reconnaissance mutuelle aux sanctions pécuniaires, 2005/214/JAI, p. 0016.

14.. JO L 196 02.08.2003 p. 0045.

15.. Pour un point de vue synthétique sur ces mesures, voir : Communication sur la reconnaissance mutuelle des décisions judiciaires en matière criminelle et sur le renforcement de la confiance mutuelle entre les Etats membres, 19.05.2005, COM(2005) 195 final : http://europa.eu.int/comm/justice_home/doc_centre/criminal/doc/ com_2005_195_en.pdf

16.. Art. 8 de la décision-cadre relative au mandat d'arrêt européen, op. cit.

17.. Préambule 5 de la décision-cadre relative au mandat d'arrêt européen, op. cit.

18.. Art. 3 et 4 de la décision-cadre relative au mandat d'arrêt européen, op. cit.

19.. Préambule 10 de la décision-cadre relative au mandat d'arrêt européen, op. cit., Lord Hope of Craighead, Office of the King's Prosecutor Brussels v Cando Armas \& ors, [2005] UKHL 67, (2005) 1 WLR 1389, (2005) 2 All ER 181 § 23. 
20.. Communication sur la reconnaissance mutuelle des décisions judiciaires en matière criminelle et sur le renforcement de la confiance mutuelle entre les Etats membres, 19 mai 2005, $\operatorname{COM(2005)~} 195$ final, § 18, voir note 15.

21.. L'intitulé exact de cette convention est « Convention européenne de sauvegarde des droits de l'Homme et des Libertés fondamentales ». Disponible sur : http:// www.echr.coe.int/echr.

22. Proposition de décision-cadre relative à certains droits procéduraux accordés dans le cadre des procédures pénales dans l'Union européenne, 28 avril 2004, COM(2004) 328/final : http://europa.eu.int/eurlex/lex/LexUriServ/LexUriServ.do?uri=CELEX: 52004PC0328:FR:NOT.

23.. Pour un résumé des mesures discutées et publiées dans le Livre vert, voir : Communication sur la reconnaissance mutuelle des décisions judiciaires en matière criminelle et sur le renforcement de la confiance mutuelle entre les Etats membres, op. cit.

24.. Alegre S., Leaf M., European Arrest Warrant - A Solution Ahead of its Time? London, JUSTICE, 2003.

25.. [2005] EWHC 915 (Admin), non mentionné, http://www.bailii.org/ew/cases/EWHC/ Admin/2005/915.html

26.. Afin de respecter les caractéristiques des différents systèmes pénaux, les noms et niveaux des autorités judiciaires ne seront pas traduits. Dans le système anglais et irlandais, les High Courts représentent le niveau d'appel des cours judiciaires et administratives françaises.

27. Case 1 AK 4/05, Strafverteidiger 2005, 232.

28. [2005] UKHL 67, (2005) 1 WLR 1389, (2005) 2 All ER 181, § 27-30.

29.. [2006] EWHC 167 (Admin), non mentionné, § 30 et 41, disponible sur http:// www.bailii.org/ew/cases/EWHC/Admin/2006/167.html

30.. [2005] IEHC 321 at p. 5, disponible sur http://www.bailii.org/ie/cases/IEHC/ 2005/321.html

31.. Case 3 Ausl 102/2004, Neue Zeitschrift für Strafrecht 2005, 348

32. [2005] IEHC 284 at p. 4 disponible sur http://www.bailii.org/ie/cases/IEHC/ 2005/284.html

33.. [2005] EWHC 3036 (Admin), non mentionné.

34.. S 21 de l'English Extradition Act 2003 ; S 37 de l'Irish European Arrest Warrant Act 2003.

35.. Voir note 25 , § 24 .

36.. [2006] EWHC 299 (Admin), non mentionné, §111 :http://www.bailii.org/ew/cases/ EWHC/Admin/2006/200.html

37. Voir note 27, § 51.

38.. [2005] EWHC 2526 (Admin), non mentionné.

39.. [2005] EWHC 2932 (Admin), non mentionné : http://www.bailii.org/ew/cases/

EWHC/Admin/2005/2931.html

40.. [2002] EWHC 1278 (Admin), non mentionné, § 22, 27 : http://www.bailii.org/ew/ cases/EWHC/Admin/2002/1278.html

41.. [2005] IEHC 386, non mentionné, p. 4 : http://www.bailii.org/ie/cases/IEHC/ 2005/386.html

42.. Premier Rapport de la session 2004-05, HL Papier 28, § 6 disponible sur : http:// www.publications.parliament.uk/pa/ld200405/ldselect/ldeucom/28/28.pdf. « A national judge may have no choice but to enforce the order of a court of another 
member state without himself examining the facts and merely on the basis of a form containing a number of boxes that have been ticked. For such a system to be acceptable there must be confidence that the individual, the subject of the proceedings, has been and will be treated fairly. Compliance by member states with minimum procedural standards for criminal investigations and prosecutions is, therefore, essential ».

43.. Voir note $25, \S 5$.

44.. David Irving, historien britannique, est connu pour ses thèses négationnistes et antisémites.

45.. Initiative de la république d'Autriche, de la république de Finlande et du royaume de Suède en vue d'adopter la décision du Conseil relative au renforcement de l'entraide en matière d'arrestation et de transfert des personnes entre les Etats membres, $\mathrm{JO}^{\circ} \mathrm{C}$ 150, 21 juin 2005, p. 0001. Disponiblesur : http://www.eurowarrant.net/documents/ cms_eaw_235_1_OJC.150.2005.p1.pdf

46.. Jugement du 13 juillet 2005, C-303/05 : http://www.eurowarrant.net/documents/ cms_eaw_219_1_CourdArb.Belgique.3027fr.pdf

47.. Pour une analyse des difficultés et des approximations des systèmes judiciaires des Etats membres, voir Weyembergh S., Approximation of Criminal Laws, the Constitutional Treaty and the Hague Programme, 2005, 42 CMLR 1567.

\section{RÉSUMÉS}

La reconnaissance mutuelle des décisions judiciaires constitue la pierre angulaire de la coopération pénale européenne. Philosophie officielle de l'Union européenne, ce principe se trouve à la base des instruments de coopération tels le mandat d'arrêt européen. Les premiers arrêts rendus par les Cours anglaises, irlandaises et allemandes démontrent cependant qu'une absence de confiance en les Etats membres de l'UE au niveau des systèmes pénaux gêne considérablement la mise en pratique de cette philosophie. Cet article évalue la jurisprudence émergente sur cette question, dans l'optique de déterminer à la fois l'efficacité des instruments actuels de reconnaissance mutuelle et de mettre en évidence les difficultés inhérentes à ce principe concernant la protection des droits fondamentaux. L'auteur conclut sur la nécessité d'une certaine harmonisation des droits pénaux des Etats membres pour que la confiance mutuelle puisse être à la base du principe de reconnaissance, ce qui permettra de réaliser « un espace de liberté, la sécurité et la justice ».

Mutual recognition of judicial decisions is the cornerstone of European criminal justice cooperation. This is the European Union's official philosophy underlying instruments such as the European Arrest Warrant. First experiences in the courts of England, Ireland and Germany show, however, that a noticeable lack of mutual trust in EU member states' criminal justice systems considerably hampers the application of that philosophy in practice. This article assesses the emerging case-law in order to determine the effectiveness of current mutual recognitions instruments and highlights the difficulties inherent in the principle of mutual recognition when it comes to the protection of fundamental rights. It concludes that some measure of harmonisation or approximation of member states' criminal laws will be necessary if mutual 
trust as a basis for true mutual recognition is to be achieved in an "area of freedom, security and justice".

\section{INDEX}

Index géographique : Union européenne

Mots-clés : construction européenne, coopération internationale, juge, libertés publiques, police, sécurité

\section{AUTEUR}

\section{MAIK MARTIN}

Maik Martin est membre (Legal Officer) de l'organisation anglaise JUSTICE spécialisée dans les questions juridiques de respect des droits de l'Homme. Détenteur du titre d'avocat en Allemagne, il est également diplômé en droit des universités de Berlin, Durham et Oxford. 